\title{
Plasmonic Circular Nanostructure for Enhanced Light Absorption in Organic Solar Cells
}

\author{
Nan-Fu Chiu, ${ }^{1}$ Cheng-Hung Hou, ${ }^{2}$ Chih-Jen Cheng, ${ }^{1}$ and Feng-Yu Tsai ${ }^{2}$ \\ ${ }^{1}$ Institute of Electro-Optical Science and Technology, National Taiwan Normal University, No. 88, Section 4, \\ Ting-Chou Road, Taipei 11677, Taiwan \\ ${ }^{2}$ Department of Material Science and Engineering, National Taiwan University, Taipei, Taiwan
}

Correspondence should be addressed to Nan-Fu Chiu; nfchiu@ntnu.edu.tw

Received 1 February 2013; Revised 1 April 2013; Accepted 21 April 2013

Academic Editor: Fahrettin Yakuphanoglu

Copyright (C) 2013 Nan-Fu Chiu et al. This is an open access article distributed under the Creative Commons Attribution License, which permits unrestricted use, distribution, and reproduction in any medium, provided the original work is properly cited.

\begin{abstract}
This study attempts to enhance broadband absorption in advanced plasmonic circular nanostructures (PCN). Experimental results indicate that the concentric circular metallic gratings can enhance broadband optical absorption, due to the structure geometry and the excitation of surface plasmon mode. The interaction between plasmonic enhancement and the absorption characteristics of the organic materials (P3HT:PCBM and PEDOT:PSS) are also examined. According to those results, the organic material's overall optical absorption can be significantly enhanced by up to $\sim 51 \%$ over that of a planar device. Additionally, organic materials are enhanced to a maximum of $65 \%$ for PCN grating pitch $=800 \mathrm{~nm}$. As a result of the PCN's enhancement in optical absorption, incorporation of the PCN into P3HT:PCBM-based organic solar cells (OSCs) significantly improved the performance of the solar cells: short-circuit current increased from 10.125 to 12.249 and power conversion efficiency from $3.2 \%$ to $4.99 \%$. Furthermore, optimizing the OSCs architectures further improves the performance of the absorption and PCE enhancement.
\end{abstract}

\section{Introduction}

Recent studies have demonstrated the feasibility of designing metallic nanostructures to increase optical absorption in solar cells by excitation of surface plasmon polaritons (SPPs) mode [1-5]. The absorption properties of plasmonic material have also been investigated, based on multiple resonances $[6-8]$ and omnidirectional absorption $[6,9,10]$ as well as frequency dispersion engineering $[11,12]$. As electromagnetic waves at the interface between a dielectric material and a metal surface, SPPs propagate along a metal-dielectric interface with exponentially decaying into both media [1316]. For the excitation of SPPs, a previous study used circular grating structures, which alter the light momentum to achieve phase matching condition [17]. This structure has received a significant amount of interest for several applications, including the enhancement of transmitted light [18], plasmonic lens $[19,20]$, distributed feedback (DFB) lasers [21], and plasmonic antennas $[22,23]$.

This study describes the enhancement of broadband absorption in advanced plasmonic circular nanostructures
(PCN). Reflected intensity is measured at a white light source and three visible wavelengths $(488 \mathrm{~nm}, 532 \mathrm{~nm}$, and $632 \mathrm{~nm})$ by using a charge-coupled device (CCD). Owing to the rotation symmetry in circular structures, the optical absorption is enhanced in both transverse-magnetic (TM) and transverse-electric (TE) polarizations. Additionally, absorption spectra for normally incident light are evaluated by UVVis spectrophotometer. The absorption spectrum reveals the enhancement broadband absorption in concentric circular metallic gratings due to the structure geometry and the excitation of SPPs mode. Furthermore, the organic solar cells (OSCs) performance properties are investigated with respect to interaction between periodic PCN structure with organic materials, revealing an enhancement of broadband absorption and power conversion efficiency.

\section{Experiments}

Periodic PCN structures were fabricated using standard electron beam lithography and lift-off procedures [24]. In our experiments, a $2 \mathrm{~nm}$-thick $\mathrm{Cr}$ film and a $50 \mathrm{~nm}$-thick gold 


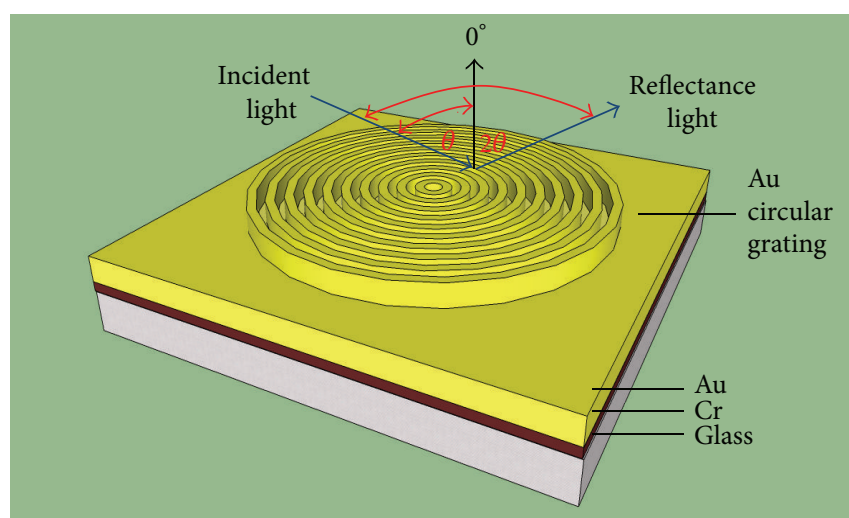

(a)

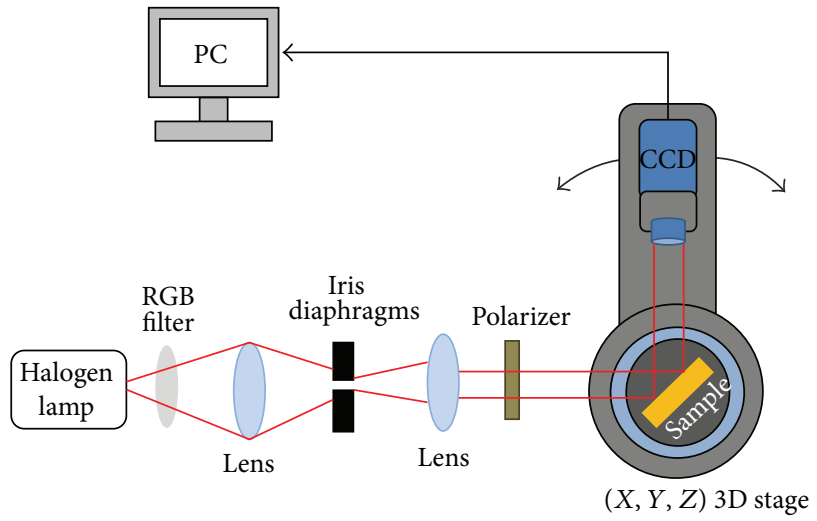

(c)

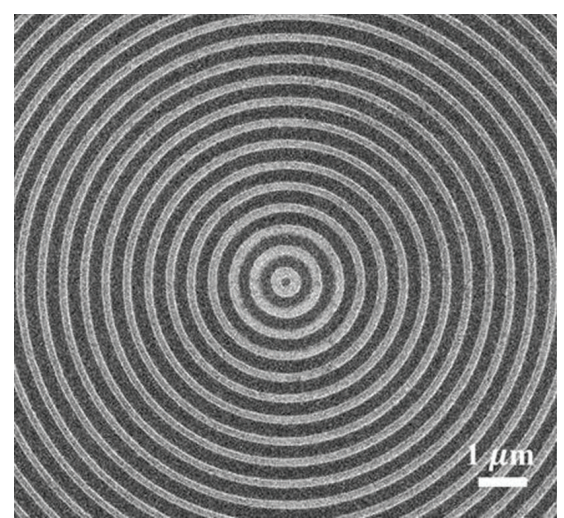

(b)

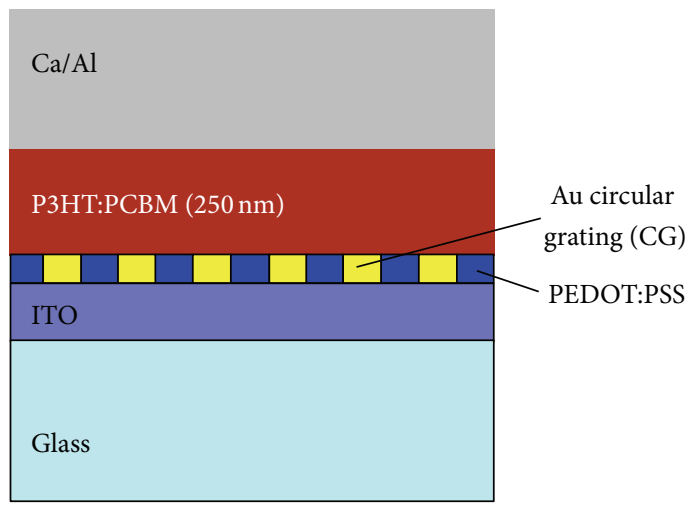

(d)

Figure 1: (a) Schematic representation of a sample for a glass substrate/Cr film (2 nm)/Au film (47 nm)/Au concentric circular grating (50 nm). (b) An SEM image of the fabricated PCN sample. The grating ring width and pitch size of the pattern are $250 \mathrm{~nm}$ and $500 \mathrm{~nm}$, respectively. (c) The optical setup for reflected intensity measurement. (d) Cross-sectional view of the thin-film OSCs architecture made of glass/ITO/Au circular grating/PEDOT:PSS/P3HT:PCBM/Ca/Al. A $50 \mathrm{~nm}$-thick PCN was fabricated on the ITO anode layer using standard electron beam lithography and lift-off procedures.

film were sequentially deposited on a glass substrate. $\mathrm{Cr}$ was used as an adhesion layer between the glass substrate and $\mathrm{Au}$ layer. The $50 \mathrm{~nm}$-thick Au concentric circular gratings were then fabricated for various periods (ranging from $400 \mathrm{~nm}$ to $800 \mathrm{~nm}$ ) and for a duty cycle of $50 \%$ on top of the first $\mathrm{Au}$ layer. The area of each circular grating was $1.2 \times 1.2 \mathrm{~mm}^{2}$. Figures 1(a) and 1(b) show a schematic representation and a scanning electron microscope (SEM) picture of the periodic PCN structure sample. The grating ring width and pitch size of the pattern are $250 \mathrm{~nm}$ and $500 \mathrm{~nm}$, respectively.

For PCN measurement systems, angular interrogation mode of reflection angle measurement system $(\theta-2 \theta)$ was used for the SPPs resonance angular absorption. Figure 1(c) illustrates the optical measurement setup. The samples were illuminated by using a white light source (halogen lamp) with red, green, and blue (RGB) filters $(488 \mathrm{~nm}, 532 \mathrm{~nm}$, and $632 \mathrm{~nm}$ ). The reflected light was then collected by using a CCD camera. Notably, the incident polarization was controlled by a linear polarizer. Moreover, absorption spectra for normally incident light by UV-Vis spectrophotometer (Hitachi U-2900) were determined.

The OSC device used for this study was fabricated as follows. Prepatterned ITO-coated glass (sheet resistance of
$15 \Omega /$ sq) substrates were ultrasonically cleaned for about $3 \mathrm{~min}$ in deionized water with $10 \%$ detergent, deionized water, acetone, and isopropanol alcohol, sequentially. A $50 \mathrm{~nm}$-thick concentric Au metal PCN was fabricated on the ITO anode layer using standard electron beam lithography and lift-off procedures. A PEDOT:PSS (Baytron P) film of $40 \mathrm{~nm}$ thickness was spin-coated at 4000 r.p.m. for $60 \mathrm{~s}$, and the resulting PEDOT:PSS layer was baked at $170^{\circ} \mathrm{C}$ for $15 \mathrm{~min}$. A $20 \mathrm{mg} / \mathrm{mL}$ 1,2-dichlorobenzene (DCB) solution of P3HT:PCBM with a 1:1 weight ratio was stirred under nitrogen atmosphere at $40^{\circ} \mathrm{C}$ for $14 \mathrm{~h}$. The active layer was obtained by spin coating the blend at 600 r.p.m. for $50 \mathrm{~s}$, and the film thickness was $\sim 250 \mathrm{~nm}$. Before cathode deposition, the films were thermally annealed at $110^{\circ} \mathrm{C}$ for $10 \mathrm{~min}$. Finally, the $\mathrm{Ca} / \mathrm{Al}$ $(20 \mathrm{~nm} / 100 \mathrm{~nm})$ were used in thermal evaporation to deposit as an electrode, as shown in Figure 1(d).

\section{Results and Discussions}

Figure 2 illustrates the reflected intensity images of a periodic PCN structure with a pitch size of $800 \mathrm{~nm}$. Absorption of the sample appears dark and colorless in reflected intensity 


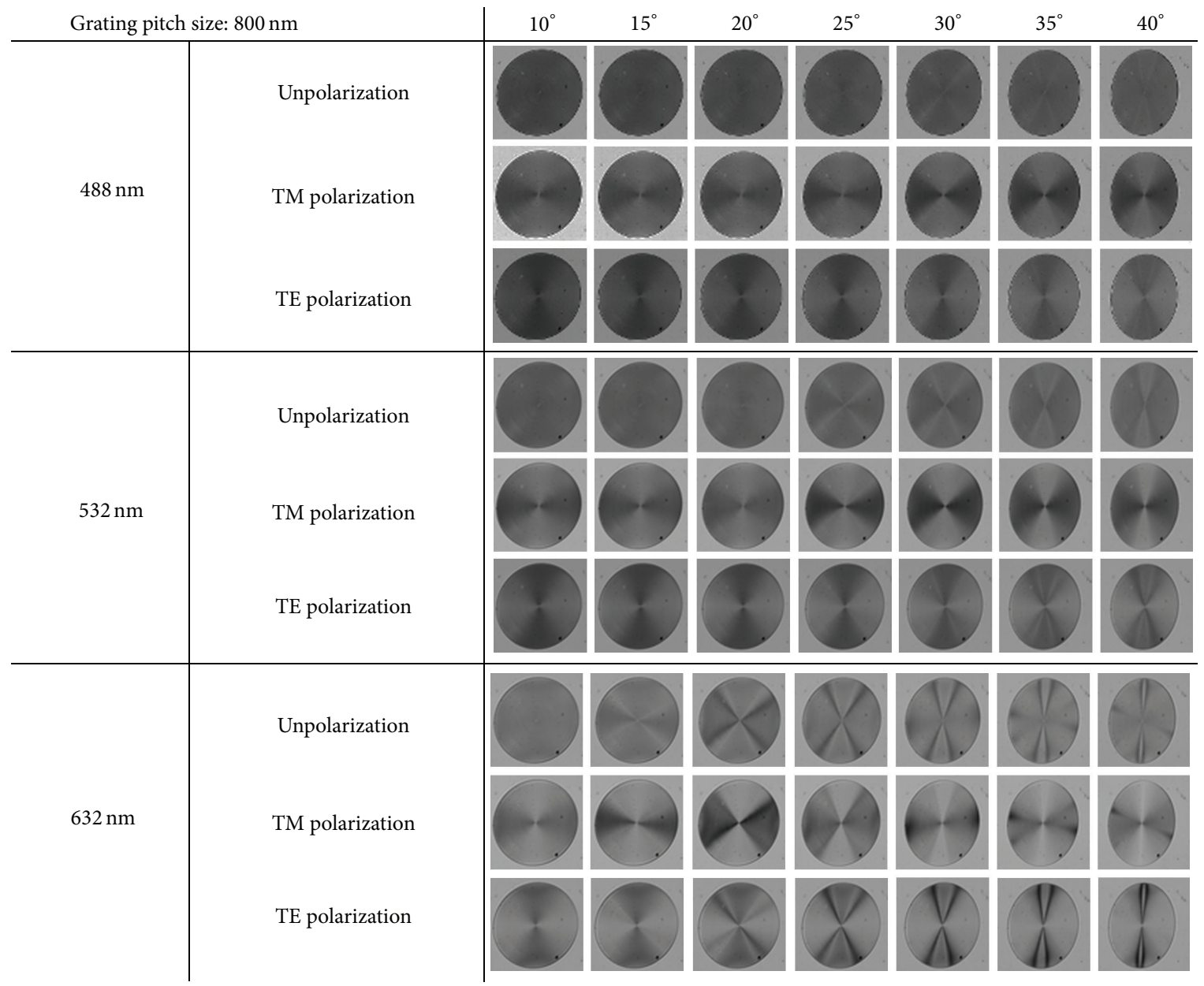

Figure 2: Measured reflected intensity images with various incident angles (ranging from $10^{\circ}$ to $40^{\circ}$ ) by CCD through TM-polarized, TEpolarized, and unpolarized incident light at three visible wavelengths of $488 \mathrm{~nm}, 532 \mathrm{~nm}$, and $632 \mathrm{~nm}$, respectively. The grating pitch size is $800 \mathrm{~nm}$.

images. According to this figure, the images vary with incident angles and incident wavelengths. Experimental results indicate that the enhancement of optical absorption is associated with polarization of the incident light. Enhancement of the optical absorption in both TM and TE polarizations is attributed to rotation symmetry in a periodic PCN structure. When the incident light is TM polarized, the optical absorption is enhanced in a horizontal direction. When the incident light is TE polarized, the optical absorption is enhanced in a vertical direction.

Figure 3 shows the normalized reflectance intensity spectra. The incident light is unpolarized. Although enhancement of the absorption depends slightly on incident angles, the absorption can be more significantly improved than that of a planar Au film without grating at a white light source and three visible wavelengths (488 nm, $532 \mathrm{~nm}$ and $632 \mathrm{~nm}$ ).

This study also attempts to more thoroughly understand how absorption enhancement and incident wavelengths are related by determining absorption spectra for normally incident light by UV-Vis spectrophotometer. In contrast to a planar Au film without grating, the absorption spectrum shows improved broadband absorption in Au concentric circular gratings, as shown in Figure 4(a). Additionally, several peaks of enhanced absorption in a spectrum are observed, due to the excitation of SPPs mode at the Au/air layer interface. As is well known, the periodic PCN structure can provide the in-plane momentum required for the incident light to excite SPPs. The SPPs excitation causes a strong electromagnetic field near the metal surface, which is expected to enhance optical absorption [13, 16, 25]. This enhancement occurs when the momentum matching condition is satisfied. The condition can be written as [16]:

$$
k_{/ /}=m \frac{2 \pi}{\Lambda}=k_{o} \sqrt{\frac{\varepsilon_{m} \varepsilon_{d}}{\varepsilon_{m}+\varepsilon_{d}}}=k_{\mathrm{sp}}
$$

where $k_{o}=\omega / c$ is a free-space wave vector; $k_{/ /}$is an inplane wave vector; $m$ is to the resonant order; $\Lambda$ is the grating pitch; $\varepsilon_{m}$ and $\varepsilon_{d}$ are the permittivities of the metal (Au) and dielectric (air), respectively. The position of the SP resonance 


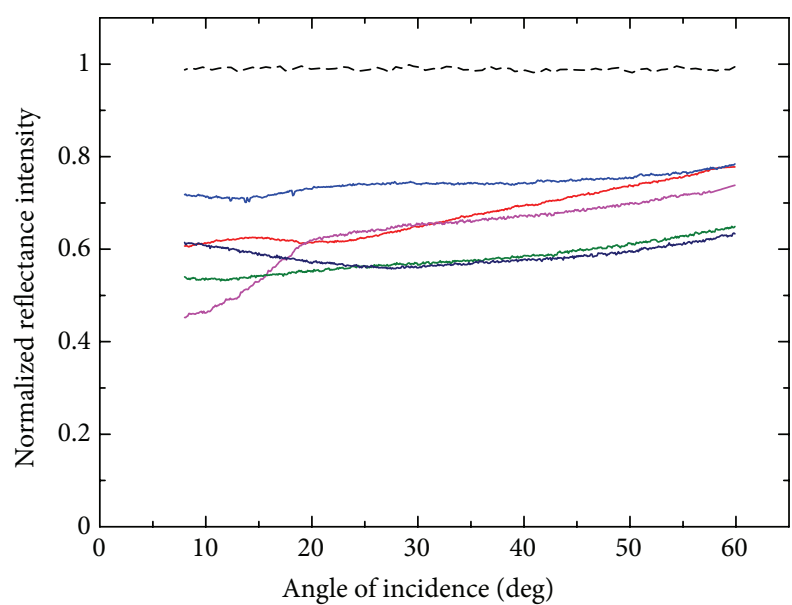

(a)

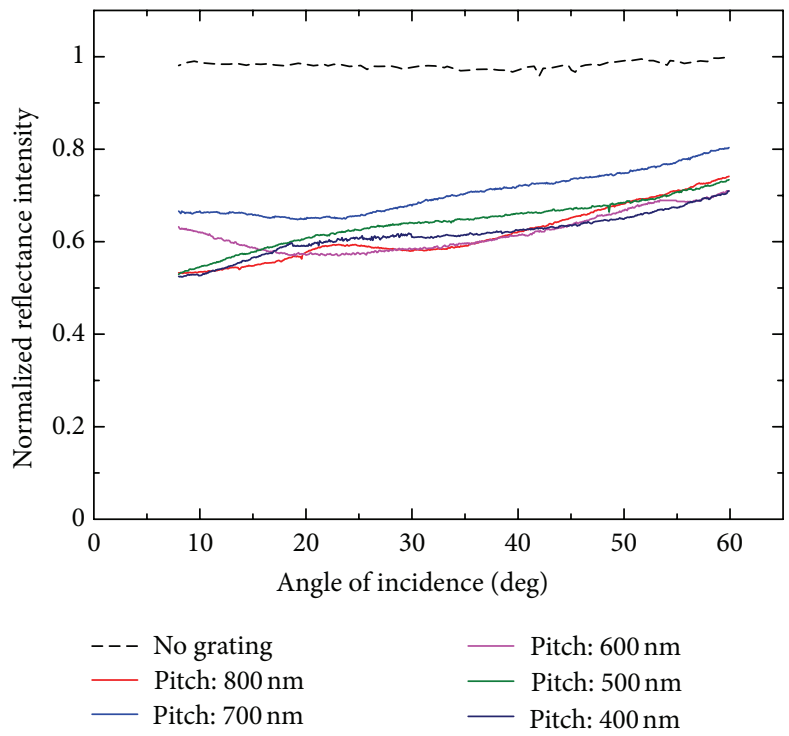

(c)

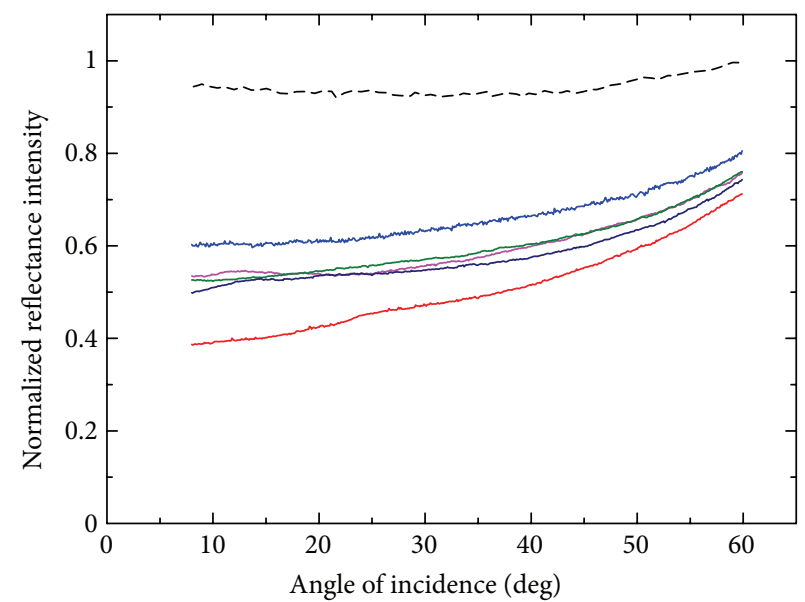

(b)

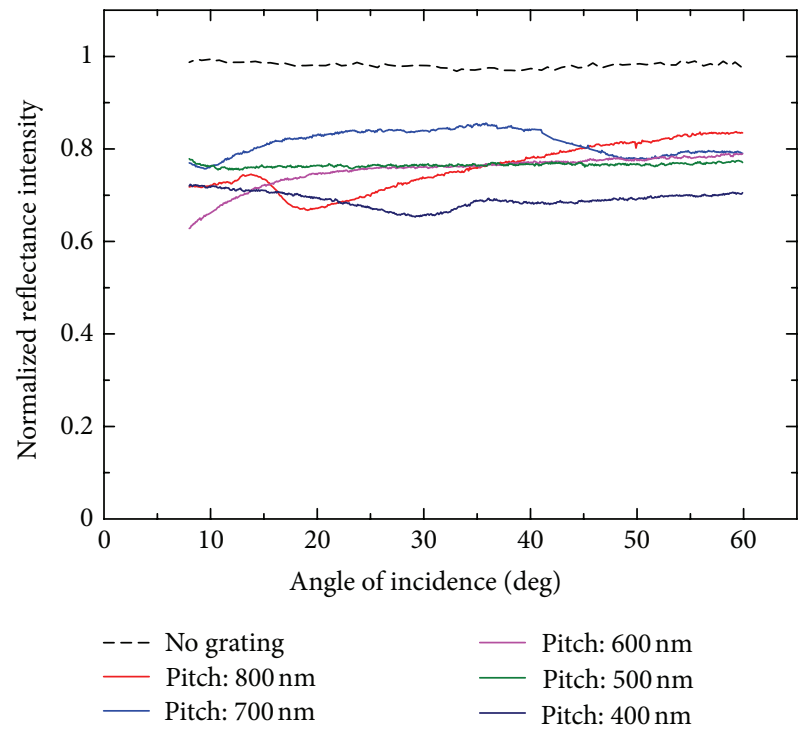

(d)

FIGURE 3: Normalized reflectance intensity as a function of incident angles for grating pitches $=400,500,600,700,800 \mathrm{~nm}$ and a planar reference sample (Au film without grating). The samples illuminated by a white light source (a) and three visible wavelengths $488 \mathrm{~nm}(\mathrm{~b})$, $532 \mathrm{~nm}(\mathrm{c})$, and $632 \mathrm{~nm}(\mathrm{~d})$, respectively. The incident light is unpolarized.

$\lambda_{\mathrm{sp}}=2 \pi / k_{o}$ for normally incident light can be expressed by the following relation [16]:

$$
\lambda_{\mathrm{sp}}=\frac{\Lambda}{m} \sqrt{\frac{\varepsilon_{m}+\varepsilon_{d}}{\varepsilon_{m} \varepsilon_{d}}} .
$$

The peaks in the spectrum are near the positions calculated by (2). The observed resonant wavelengths for each grating are $409 \mathrm{~nm}($ pitch $=400 \mathrm{~nm}), 544 \mathrm{~nm}($ pitch $=500 \mathrm{~nm}), 610 \mathrm{~nm}$ $($ pitch $=600 \mathrm{~nm}), 681 \mathrm{~nm}($ pitch $=700 \mathrm{~nm})$, and $494 \mathrm{~nm}$ (pitch $=800 \mathrm{~nm})$. Obviously, the experimental periodic PCN resonant wavelengths are close to the theoretical SPPs resonant wavelengths [26-28], as shown in Figure 4(b).

Figure 5 shows the absorption enhancement for organic materials (PEDOT:PSS/P3HT:PCBM) on a periodic PCN structure. Figure 5(a) reveals similar broadband absorption enhancement (ranging from 300 to $1100 \mathrm{~nm}$ ) in the ITO/glass substrate (dashed lines). As mentioned earlier, the periodic PCN structure can enhance broadband absorption. Therefore, the overall optical absorption in organic materials can be significantly enhanced by up to $\sim 51 \%$ over that of a planar device without grating. The absorption spectrum of the periodic PCN structure shows a greater broadband enhancement than that of the ITO glass without grating. Therefore, overall absorption of an organic layer can be significantly enhanced owing to the concentric Au metal grating. An absorption peak is also observed around $745 \mathrm{~nm}$, due to the excitation of SPPs mode at the Au/organic layer interface. Excitation of the SPPs enhances the highly localized field around the nanostructures and absorption of specific wavelengths of light. To understand this phenomenon, this study determined the absorption spectra of ITO/Au circulargrating/PEDOT:PSS/P3HT:PCBM films with various pitch Au circular gratings. According to Figure 5(b), the absorption 


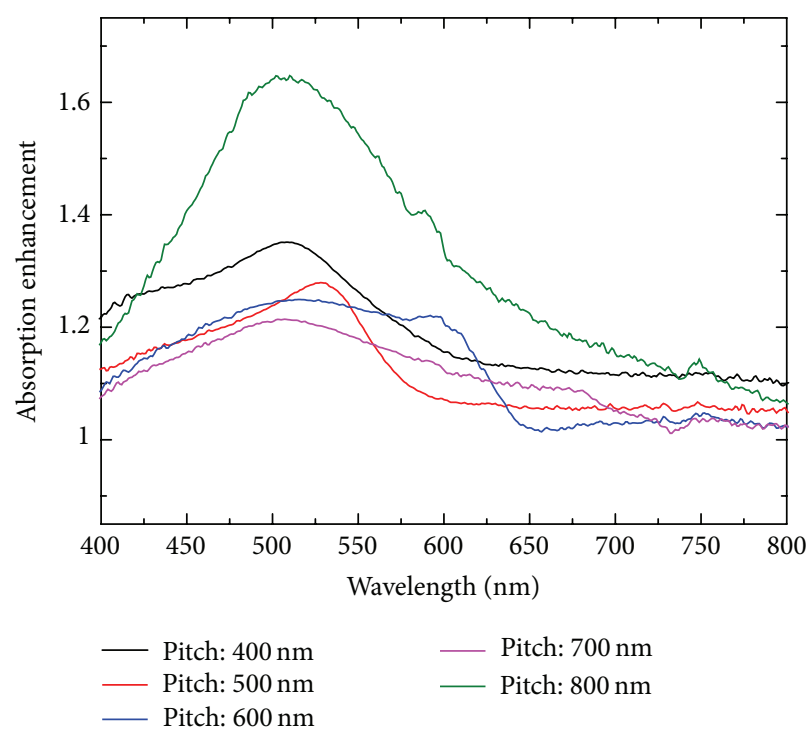

(a)

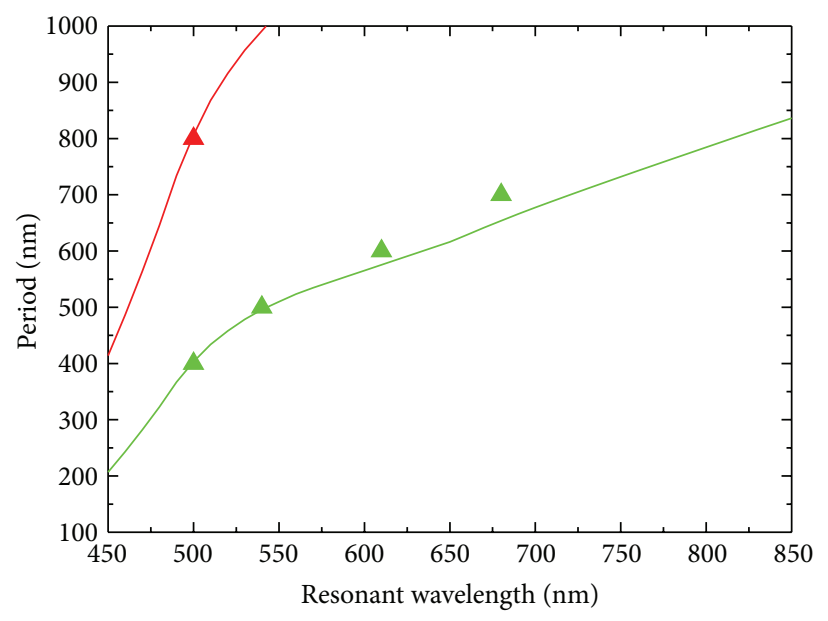

Theoretical calculation

$m=1$

$m=2$
Experimental data

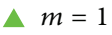

A $m=2$

(b)

Figure 4: (a) Comparison of enhanced absorption in terms of different pitch size Au concentric circular grating. (b) Experimental and theoretical (2) SP resonant wavelengths $\lambda_{\text {sp }}$ as a function of pitch.

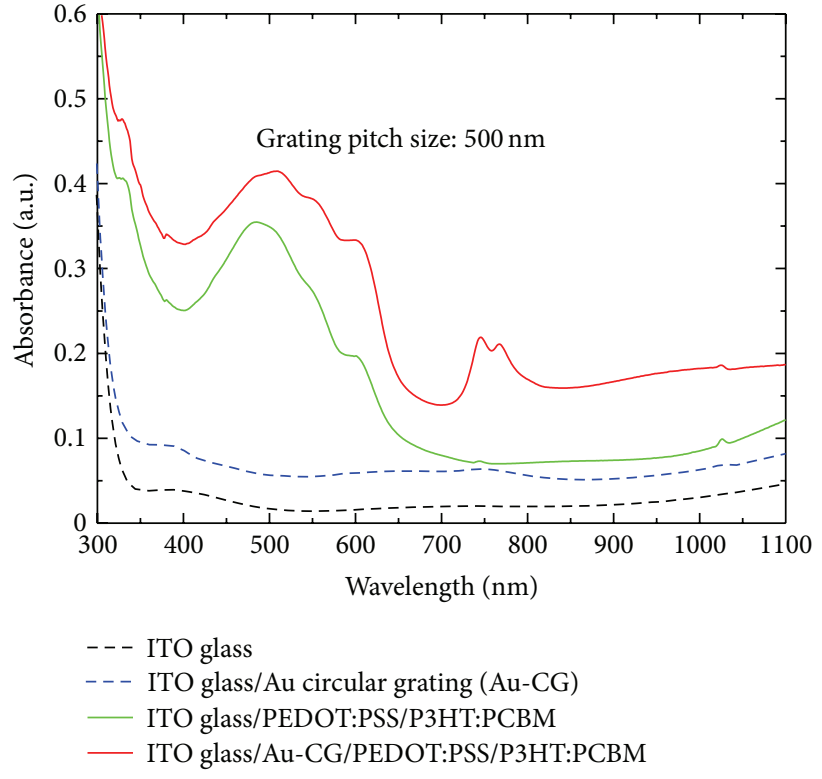

(a)

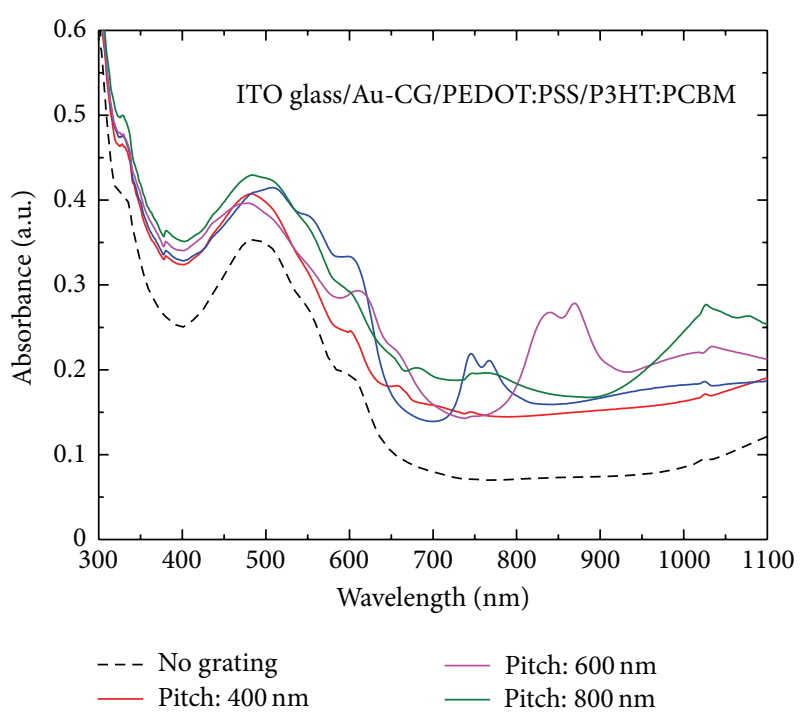

(b)

FIGURE 5: (a) Measured absorption enhancement for organic materials (PEDOT:PSS/P3HT:PCBM) on periodic PCN structure in comparison with a planar device. (b) Measured absorption enhancement for organic materials (PEDOT:PSS/P3HT:PCBM) on a periodic PCN structure with various grating pitch sizes.

increases for extended periods, owing to that the grating decreases the reflection and permits of more light to couple into the organic layer. The maximum enhancement of $\sim 65 \%$ is obtained for a grating pitch of $800 \mathrm{~nm}$. Optimization of OSC architectures further improves absorption enhancement.

Light current density-voltage (J-V) characteristics curves were taken under simulated AM1.5 illumination from a solar simulator of Oriel-Sol3A 94023A equipped with a $450 \mathrm{~W}$ Xe lamp and an AM1.5 filter (Newport Inc.) and a Kiethley 2400 source meter. The light intensity was calibrated using a reference Si solar cell of Oriel-91150 (Newport Inc.). All photoelectron measurements were taken in ambient air at room temperature. Figure 6 shows the J-V characteristics of the OSCs devices with different structures, and Table 1 summarizes their parameters. Additionally, the periodic PCN structure (ITO/Au circular grating/PEDOT:PSS/P3HT:PCBM/Ca/Al) 


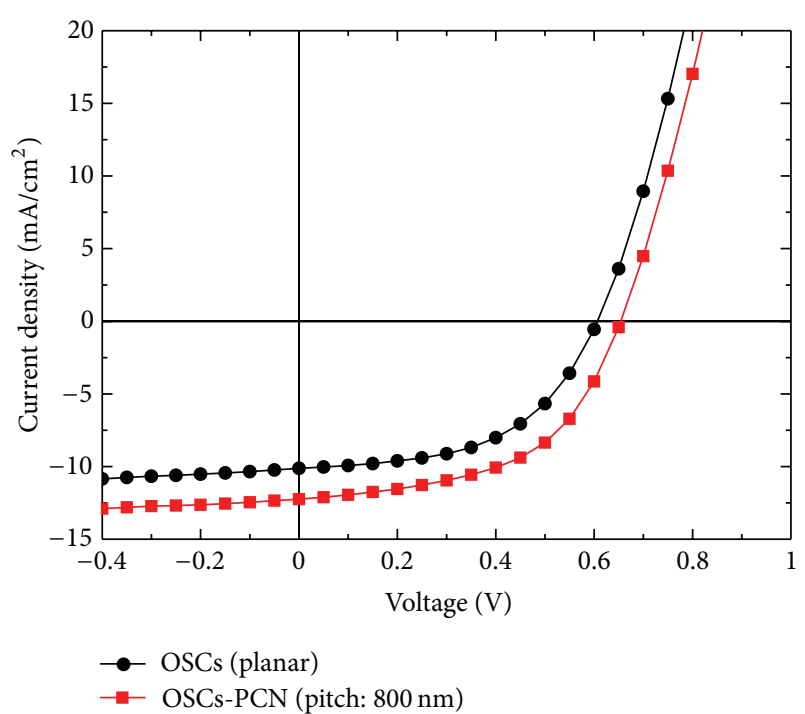

FIGURE 6: Current density versus voltage (J-V) characteristics of the OSCs on planer and periodic PCN structures. The J-V characteristics of devices: planar device (black circle), with a circular grating structure (red square), and recorded under illumination at $100 \mathrm{~mW} / \mathrm{cm}^{2}$ (AM 1.5).

TABLe 1: Photovoltaic performances of organic solar cells for planar with periodic PCN structure under AM1.5 illumination $\left(100 \mathrm{~mW} / \mathrm{cm}^{2}\right)$.

\begin{tabular}{lcccc}
\hline Device & $V_{\mathrm{oc}}(\mathrm{V})$ & $J_{\mathrm{sc}}\left(\mathrm{mA} / \mathrm{cm}^{2}\right)$ & $\mathrm{FF}(\%)$ & $\mathrm{PCE}(\%)$ \\
\hline $\begin{array}{l}\text { OSCs-PCN } \\
\text { (pitch 800 nm) }\end{array}$ & 0.65 & 12.249 & 62.79 & 4.99 \\
OSCs (planar) & 0.6 & 10.125 & 52.7 & 3.20 \\
\hline
\end{tabular}

in this study has a higher short-circuit current density $\left(J_{\mathrm{sc}}\right)$, open-circuit voltage $\left(V_{\mathrm{oc}}\right)$, fill factor $(\mathrm{FF})$, and power conversion efficiency (PCE) than those of a conventional planar device (ITO/PEDOT:PSS/P3HT:PCBM/Ca/Al). It is noted that $J_{\mathrm{sc}}$ increases from $10.125 \mathrm{~mA} / \mathrm{cm}^{2}$ to $12.249 \mathrm{~mA} / \mathrm{cm}^{2}$, and $V_{\text {oc }}$ increases from $0.6 \mathrm{~V}$ to $0.65 \mathrm{~V}$ in the periodic PCN structure device. The fill factor of the PCN structure slightly increases from $52.7 \%$ to $62.79 \%$, possibly due to the decreased resistance of the now textured PCN/ITO contact. Overall, the PCN's structure shows enhanced optical absorption in total cell power conversion efficiency from $3.2 \%$ to $4.99 \%$. Our results further demonstrate that the plasmon enhanced photo current and PCE of organic solar cells using a periodic PCN structure are superior to those of the device with conventional planar electrodes.

\section{Conclusions}

This study demonstrates the enhancement of optical absorption in both TM and TE polarizations, due to the Au periodic PCN structure rotation symmetry. Absorption spectra are also measured for a normally incident light by UVVis spectrophotometer. The absorption spectrum reveals an enhanced broadband absorption in Au periodic PCN structure, due to the relationship between the pitch size of a structure and the excitation of SPP modes. Furthermore, in the organic materials (P3HT:PCBM), optical absorption in PCN nanostructures is a highly promising means of increasing absorption by $51 \%$ over that of a planar device. In terms of the J-V characteristics of the devices, the power conversion efficiencies (PCE) are $3.2 \%$ and $4.99 \%$ for a planar device and a circular nanostructure, respectively. This novel approach for plasmonic circular nanostructure improves the performance of OSCs.

\section{Acknowledgments}

The authors would like to thank the National Science Council Taiwan (Contract nos. NSC 100-2325-B-182-007 and NSC 99-2218-E-003-002-MY3) and the Emerging Research and Development Projects of National Taiwan Normal University (Contract no. NTNU100-D-01) for partially supporting this research. Ted Knoy is appreciated for his editorial assistance.

\section{References}

[1] C. Min, J. Li, G. Veronis, J. Y. Lee, S. Fan, and P. Peumans, "Enhancement of optical absorption in thin-film organic solar cells through the excitation of plasmonic modes in metallic gratings," Applied Physics Letters, vol. 96, no. 13, Article ID 133302, 2010.

[2] H. Y. Lin, Y. Kuo, C. Y. Liao, C. C. Yang, and Y. W. Kiang, "Surface plasmon effects in the absorption enhancements of amorphous silicon solar cells with periodical metal nanowall and nanopillar structures," Optics Express, vol. 20, no. 1, pp. A104-A118, 2012.

[3] M. A. Sefunc, A. K. Okyay, and H. V. Demir, "Plasmonic backcontact grating for P3HT:PCBM organic solar cells enabling strong optical absorption increased in all polarizations," Optics Express, vol. 19, no. 15, pp. 14200-14209, 2011.

[4] K. Tvingstedt, N. K. Persson, O. Inganäs, A. Rahachou, and I. V. Zozoulenko, "Surface plasmon increase absorption in polymer photovoltaic cells," Applied Physics Letters, vol. 91, no. 11, Article ID 113514, 3 pages, 2007.

[5] C. Rockstuhl, S. Fahr, and F. Lederer, "Absorption enhancement in solar cells by localized plasmonpolaritons," Journal of Applied Physics, vol. 104, no. 12, Article ID 123102, 7 pages, 2008.

[6] Y. Q. Ye, Y. Jin, and S. He, "Omnidirectional, polarizationinsensitive and broadband thin absorber in the terahertz regime," Journal of the Optical Society of America B, vol. 27, no. 3, pp. 498-504, 2010.

[7] J. Grant, Y. Ma, S. Saha, A. Khalid, and D. R. S. Cumming, "Polarization insensitive, broadband terahertz metamaterial absorber," Optics Letters, vol. 36, no. 17, pp. 3476-3478, 2011.

[8] C. Wu and G. Shvets, "Design of metamaterial surfaces with broadband absorbance," Optics Letters, vol. 37, no. 3, pp. 308310, 2012.

[9] M. Wang, C. Hu, M. Pu et al., "Truncated spherical voids for nearly omnidirectional optical absorption," Optics Express, vol. 19, no. 21, pp. 20642-20649, 2011.

[10] M. Pu, M. Wang, C. Hu et al., "Engineering heavily doped silicon for broadband absorber in the terahertz regime," Optics Express, vol. 20, no. 3, pp. 2246-2254, 2012. 
[11] K. B. Alici, A. B. Turhan, C. M. Soukoulis, and E. Ozbay, "Optically thin composite resonant absorber at the near-infrared band: a polarization independent and spectrally broadband configuration," Optics Express, vol. 19, no. 15, pp. 14260-14267, 2011.

[12] Q. Feng, M. Pu, C. Hu, and X. Luo, "Engineering the dispersion of metamaterial surface for broadband infrared absorption," Optics Express, vol. 37, no. 11, pp. 2133-2135, 2012.

[13] H. Raether, Surface Plasmons on Smooth and Rough Surface and on Gratings, Springer, Berlin, Germany, 1988.

[14] A. Otto, "Excitation of nonradiative surface plasma waves in silver by the method of frustrated total reflection," Zeitschrift für Physik, vol. 216, no. 4, pp. 398-410, 1968.

[15] E. Kretschmann, "The determination of the optical constants of metals by excitation of surface plasmons," Zeitschrift für Physik, vol. 241, no. 4, pp. 313-324, 1971.

[16] W. L. Barnes, A. Dereux, and T. W. Ebbesen, "Surface plasmonsubwavelength optics," Nature, vol. 424, pp. 824-830, 2003.

[17] X. F. Li, S. F. Yu, and A. Kumar, "Excitation and optimization modeling of surface plasmon polaritons in a concentric circular metallic grating film," Plasmonics, vol. 5, no. 1, pp. 69-74, 2010.

[18] H. J. Lezec, A. Degiron, E. Devaux et al., "Beaming light from a subwavelength aperture," Science, vol. 297, no. 5582, pp. 820822, 2002.

[19] W. Chen, D. C. Abeysinghe, R. L. Nelson, and Q. Zhan, "Plasmonic lens made of multiple concentric metallic rings under radially polarized illumination," Nano Letters, vol. 9, no. 12, pp. 4320-4325, 2009.

[20] F. Hao, R. Wang, and J. Wang, "A design method for a micron-focusing plasmonic lens based on phase modulation," Plasmonics, vol. 5, no. 4, pp. 405-409, 2010.

[21] X. F. Li and S. F. Yu, "Static and dynamic modeling of circular grating-coupled distributed feedback lasers," IEEE Journal of Quantum Electronics, vol. 44, no. 8, pp. 424-432, 1996.

[22] H. Aouani, O. Mahboud, N. Bonod et al., "Bright unidirectional fluorescence emission of molecules in a nanoaperture with plasmonic corrugations," Nano Letters, vol. 11, no. 2, pp. 637644, 2011.

[23] H. Aouani, O. Mahboub, E. Devaux, H. Rigneault, T. W. Ebbesen, and J. Wenger, "Plasmonic antennas for directional sorting of fluorescence emission," Nano Letters, vol. 11, no. 6, pp. 2400-2406, 2011.

[24] N. F. Chiu, C. W. Lin, J. H. Lee, C. H. Kuan, K. C. Wu, and C. K. Lee, "Enhanced luminescence of organic/metal nanostructure for grating coupler active long-range surface plasmonic device," Applied Physics Letters, vol. 91, no. 8, Article ID 083114, 3 pages, 2007.

[25] D. M. Schaadt, B. Feng, and E. T. Yu, "Enhanced semiconductor optical absorption via surface plasmon excitation in metal nanoparticles," Applied Physics Letters, vol. 86, no. 6, Article ID 063106, 3 pages, 2005.

[26] V. E. Ferry, M. A. Verschuuren, H. B. T. Li et al., "Light trapping in ultrathin plasmonic solar cells," Optics Express, vol. 18, no. 13, pp. A237-A245, 2010.

[27] N. Kalfagiannis, P. G. Karagiannidis, C. Pitsalidis et al., "Plasmonic silver nanoparticles for improved organic solar cells," Solar Energy Materials and Solar Cells, vol. 104, pp. 165-174, 2012.

[28] M. A. Green and S. Pillai, "Harnessing plasmonics for solar cells," Nature Photonics, vol. 6, no. 3, pp. 130-132, 2012. 

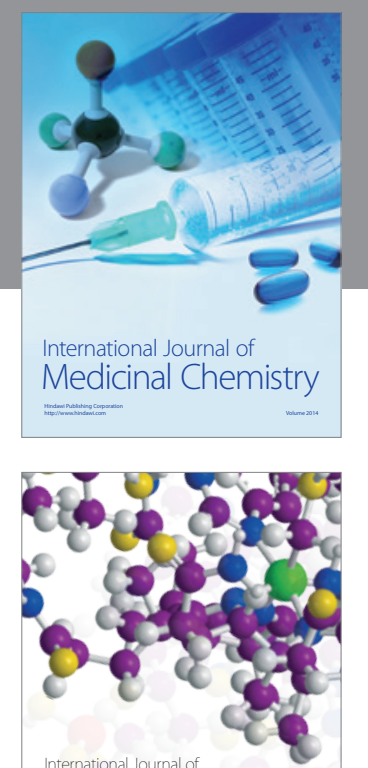

\section{Carbohydrate} Chemistry

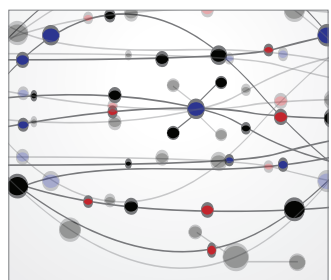

The Scientific World Journal
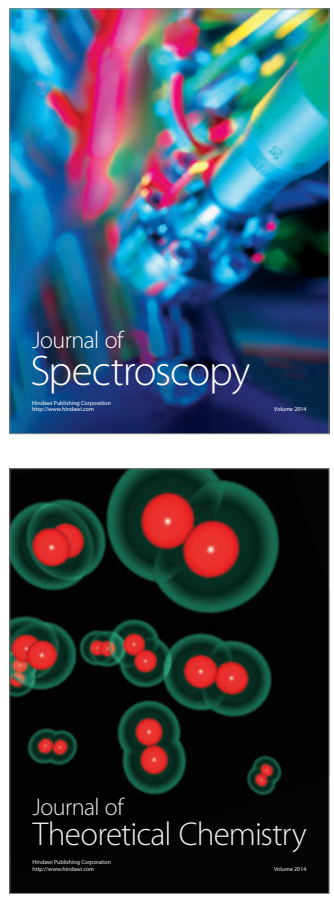
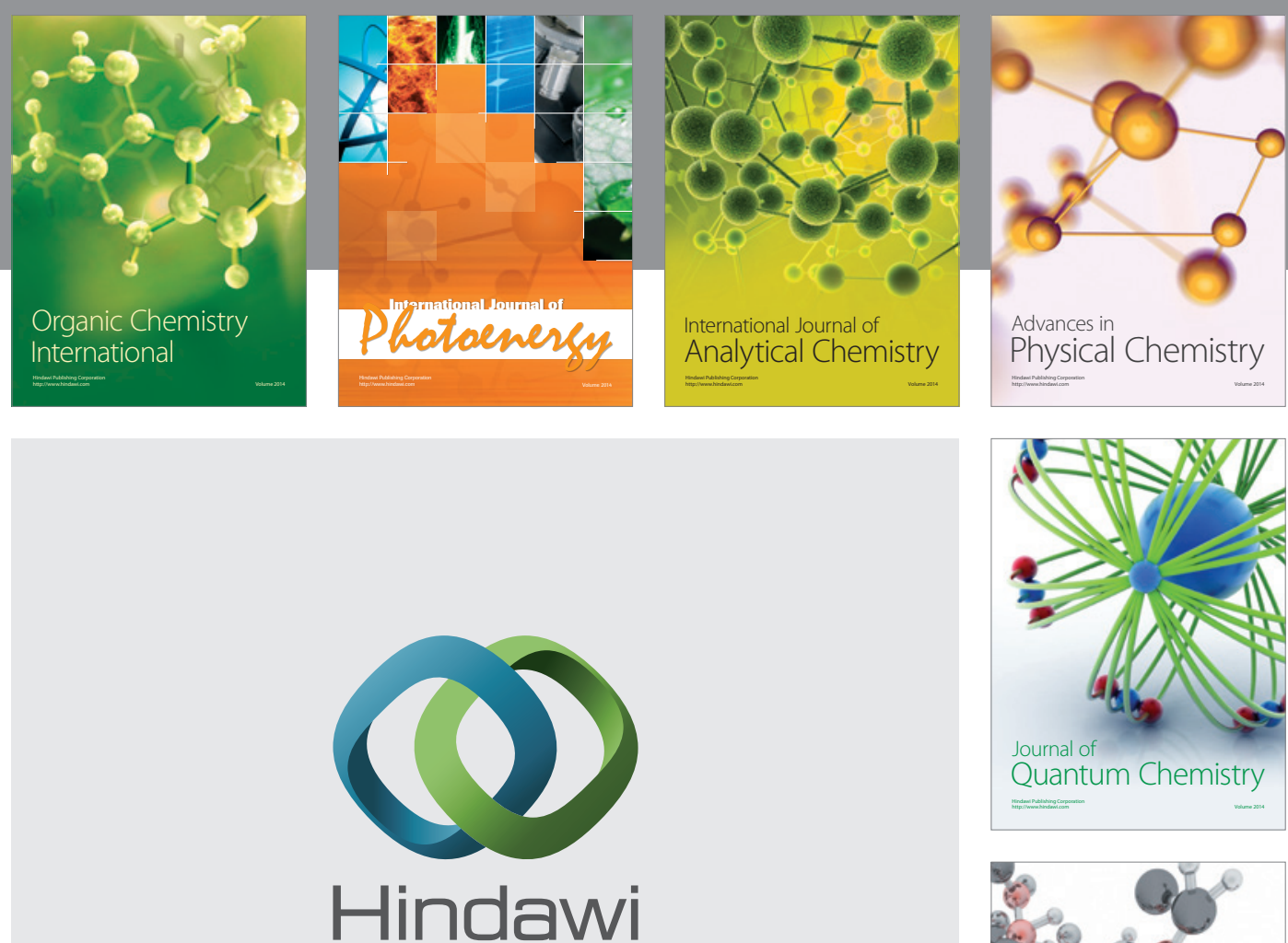

Submit your manuscripts at

http://www.hindawi.com

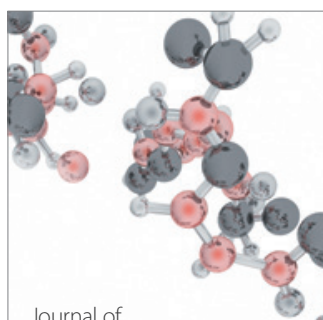

Analytical Methods

in Chemistry

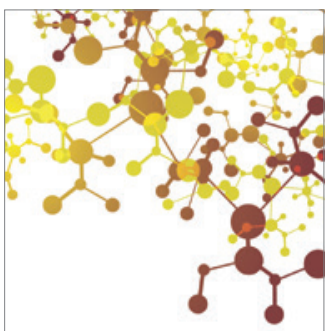

Journal of

Applied Chemistry

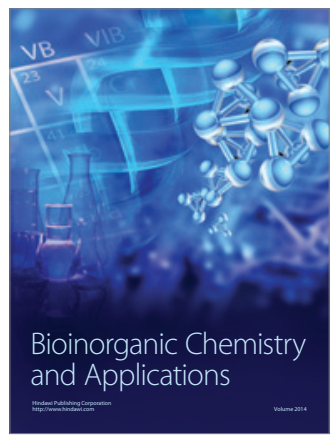

Inorganic Chemistry
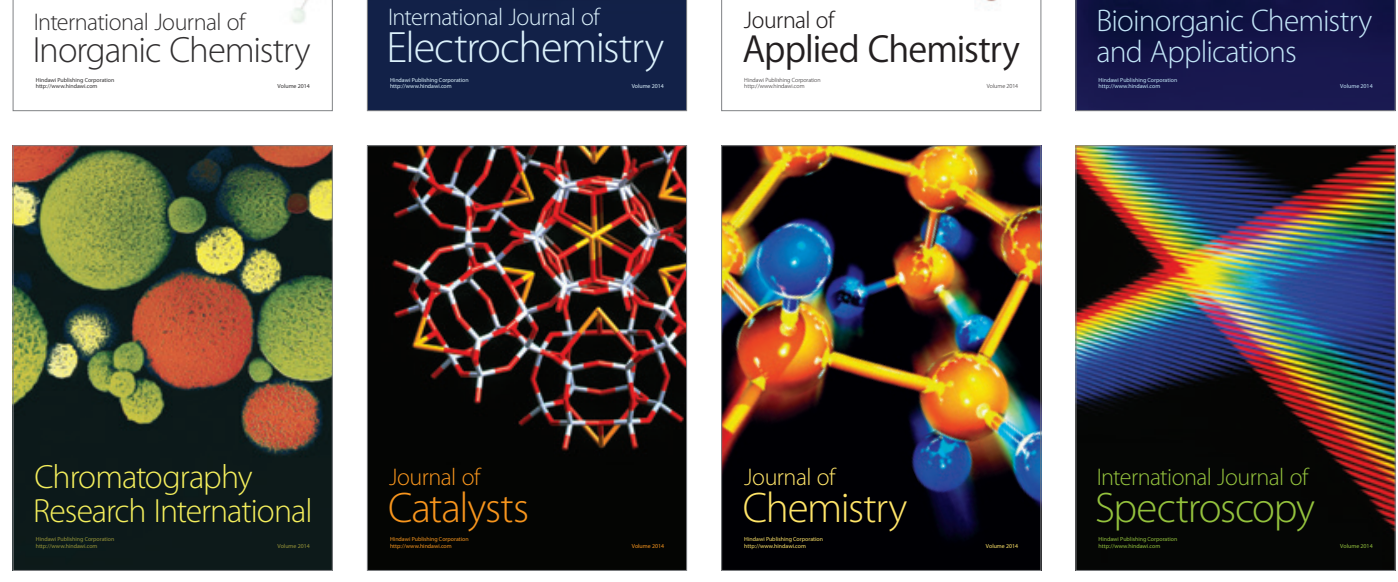